TRANSACTIONS OF THE

AMERICAN MATHEMATICAL SOCIETY

Volume 363, Number 10, October 2011, Pages 5111-5129

S 0002-9947(2011)05111-1

Article electronically published on May 4, 2011

\title{
COVARIANT DERIVATIVES OF THE BEREZIN TRANSFORM
}

\author{
MIROSLAV ENGLIŠ AND RENATA OTÁHALOVÁ
}

\begin{abstract}
Improving upon recent results of Coburn, Xia, Li, Engliš and Zhang, Bommier-Hato, and others, we give estimates for higher-order covariant derivatives of the Berezin transform of bounded linear operators on a reproducing kernel Hilbert space of holomorphic functions. The answer turns out to involve the curvature of the Bergman-type metric associated to the reproducing kernel.
\end{abstract}

\section{INTRODUCTION}

For a domain $\Omega \subset \mathbf{C}^{n}$, denote by $\mathcal{O}(\Omega)$ the vector space of all holomorphic functions on $\Omega$, and let $\mathcal{H} \subset \mathcal{O}(\Omega)$ be an arbitrary Hilbert space which has a reproducing kernel, i.e. such that the point evaluation functionals $f \mapsto f(z)$ are continuous from $\mathcal{H}$ into $\mathbf{C}$ for any $z \in \Omega$. The reproducing kernel $K(z, w)$ of $\mathcal{H}$ is then a function on $\Omega \times \Omega$, holomorphic in $z, \bar{w}$, which has the reproducing property

$$
f(z)=\left\langle f, K_{z}\right\rangle \quad \forall f \in \mathcal{H},
$$

where $K_{z}=K(\cdot, z) \in \mathcal{H}$. We will assume throughout that $\left\|K_{z}\right\|^{2}=K(z, z)$ satisfies

$$
K(z, z)>0 \quad \forall z \in \Omega .
$$

The formula

$$
K(z, z)=\left\|K_{z}\right\|^{2}=\sup \left\{|f(z)|^{2}: f \in \mathcal{H},\|f\| \leq 1\right\}
$$

then exhibits $\log K(z, z)$ as a supremum of logarithms of moduli of holomorphic functions, implying that $\log K(z, z)$ is plurisubharmonic. In other words, the matrix of mixed second-order derivatives

$$
g_{j \bar{k}}:=\frac{\partial^{2}}{\partial z_{j} \partial \bar{z}_{k}} \log K(z, z)
$$

defines an Hermitian (semi-)Riemannian metric on $\Omega$ by

$$
\|v\|_{z}^{2}:=\sum_{j, k} g_{j \bar{k}} v_{j} \bar{v}_{k}
$$

for $v \in T_{z} \Omega \cong \mathbf{C}^{n}$ the tangent space at $z \in \Omega$, which in turn induces the (semi-) distance function $\beta(\cdot, \cdot)$ on $\Omega$ in the standard way [19], 20].

Received by the editors May 16, 2008 and, in revised form, May 17, 2009.

2000 Mathematics Subject Classification. Primary 47B32; Secondary 32A36, 53B35, 32 Q15.

Key words and phrases. Berezin transform, Berezin symbol, covariant derivative, curvature, reproducing kernel.

This research was supported by GA AV ČR grant no. IAA100190802 and Ministry of Education research plan no. MSM4781305904.

(C)2011 American Mathematical Society Reverts to public domain 28 years from publication 
In his quantization program in the 1970s, Berezin [4 introduced a general symbol calculus for linear operators on reproducing kernel spaces. More specifically, for $X \in \mathcal{B}(\mathcal{H})$, the algebra of all bounded linear operators on $\mathcal{H}$, the Berezin symbol (or Berezin transform) of $X$ is the function on $\Omega$ defined as

$$
\widetilde{X}:=\left\langle X k_{z}, k_{z}\right\rangle
$$

where

$$
k_{z}=K(z, z)^{-1 / 2} K(\cdot, z)=\frac{K_{z}}{\left\|K_{z}\right\|}
$$

is the normalized kernel function at $z$. It is immediate that $\widetilde{X}$ is real analytic and $\|\widetilde{X}\|_{\infty} \leq\|X\|$, and it is well known that $X$ is uniquely determined by $\widetilde{X}$.

The prototypes of the spaces $\mathcal{H}$ are the Bergman spaces $A^{2}(\Omega)$ of all holomorphic functions in $L^{2}(\Omega, d V)$ on a bounded domain $\Omega \subset \mathbf{C}^{n}$ with Lebesgue measure $d V$, or the Segal-Bargmann(-Fock) spaces $A^{2}\left(\mathbf{C}^{n}\right)$ of all entire functions in $L^{2}\left(\mathbf{C}^{n}, d \mu\right)$ for the Gaussian measure

$$
d \mu(z)=(2 \pi)^{-n} e^{-|z|^{2} / 2} d V(z) .
$$

The reproducing kernel $K(z, w)$ is then just the original kernel function of Bergman [5] for $\Omega$ bounded, while $K(z, w)=e^{z \cdot \bar{w} / 2}$ for $\Omega=\mathbf{C}^{n}$. Similarly, the metric (1) is the Bergman metric on $\Omega \varsubsetneqq \mathbf{C}^{n}$ and coincides (up to a constant factor) with the Euclidean metric for $\Omega=\mathbf{C}^{n}$. In both cases, Coburn [11] obtained a Lipschitz estimate for the Berezin symbol on $A^{2}(\Omega)$, namely 1

$$
|\widetilde{X}(a)-\tilde{X}(b)| \leq 2\|X\| \beta(a, b)
$$

for any $a, b \in \Omega$ and $X \in \mathcal{B}\left(A^{2}(\Omega)\right)$. Furthermore, he showed in 12 that the above estimate is sharp in the sense that

$$
\sup _{\substack{a, b \in \Omega, a \neq b, 0 \neq X \in \mathcal{B}\left(A^{2}(\Omega)\right)}} \frac{|\widetilde{X}(a)-\tilde{X}(b)|}{\|X\| \beta(a, b)}=2 .
$$

It was subsequently noted by Xia (unpublished) that for $\Omega=\mathbf{C}^{n}$, the proof in [11] can even be used to provide a stronger result: namely, $\widetilde{X}$ and its partial derivatives of all orders are bounded. The present author and G. Zhang 15 improved upon and extended Xia's result by showing that $L \widetilde{X}$ is bounded for any invariant linear differential operator $L$ on $\Omega$ and any $X \in \mathcal{B}(\mathcal{H})$, when $\mathcal{H}$ is any one of the standard weighted Bergman spaces on a bounded symmetric domain $\Omega$.

The proof in [15] relied on the homogeneity of $\Omega$ under its group of holomorphic automorphisms and made it clear that the invariant geometry of $\Omega$ was, at least for bounded symmetric domains, the right context in which to view $\widetilde{X}$; for this reason, there was also stated a conjecture there to the effect that, for any $k=1,2, \ldots$,

$$
\sup _{z \in \Omega}\left\|\nabla^{k} \widetilde{X}(z)\right\|_{z} \leq c_{k}\|X\| \quad \forall X \in \mathcal{B}\left(A^{2}(\Omega)\right)
$$

with some constants $c_{k}$, for any bounded domain $\Omega \subset \mathbf{C}^{n}$. Here $\nabla^{k} \widetilde{X}$ stands for the $k$-th covariant derivative of $\widetilde{X}$, and $\|\cdot\|_{z}$ for its (tensor) norm at $z \in \Omega$ with respect to the Bergman metric (1).

\footnotetext{
${ }^{1}$ The constant 2 appears here instead of $\sqrt{2}$ in [11 due to a different normalization of the metric (2): the one used in 11 is twice our (2).
} 
For $k=1$, the above conjecture was settled in the affirmative by Coburn and Li [13, who showed that the directional derivative in the direction of $v \in \mathbf{C}^{n}$ satisfies

$$
\left\|D_{v} \tilde{X}(z)\right\| \leq 2\|X\|\|v\|_{z},
$$

implying that (5) holds for $k=1$ with $c_{1}=2$. For $k \geq 2$, the conjecture remained open.

In a different direction, H. Bommier-Hato studied the case of $\mathcal{H}=A^{2}\left(\mathbf{C}^{n}, d \mu_{m}\right)$, the space of all entire functions on $\mathbf{C}^{n}$ square-integrable with respect to the "powerGaussian" measures

$$
d \mu_{m}(z)=e^{-|z|^{m}} d V(z)
$$

on $\mathbf{C}^{n}$, with an arbitrary $m>0$. It was proved in [8] that $\widetilde{X}$ is locally Lipschitz; more specifically,

$$
|\widetilde{X}(a)-\widetilde{X}(b)| \leq C\|X\||a|^{\frac{m}{2}-1}|b-a|
$$

for $|a|$ large and $b$ in a small neighbourhood of $a$. Similarly, in 9 ] it was shown that the directional derivatives satisfy

$$
\left|D_{v} \widetilde{X}(a)\right| \leq C\|X\||a|^{\frac{m}{2}-1}\|v\|,
$$

implying that $\widetilde{X}$ is even globally Lipschitz for $m \leq 2$. Her proof went by highly technical computations using an explicit formula for the reproducing kernel in terms of a certain special function (the Mittag-Leffler function with parameter $2 / \mathrm{m}$ ), and also provided similar estimates for higher-order derivatives.

The Lipschitz estimate (3) means, in particular, that $\widetilde{X}$ is uniformly continuous with respect to the Bergman metric; this was applied for $\Omega=\mathbf{D}$, the unit disc, by Suárez [28], and for $\Omega$ the unit ball of $\mathbf{C}^{n}, n>1$, by Nam, Zheng and Zhong 25], in the study of Toeplitz algebras.

In this paper, we, first of all, extend the Lipschitz and directional derivative estimates (3), (4), (6) to arbitrary reproducing kernel Hilbert spaces $\mathcal{H}$ of holomorphic functions on $\Omega$; this covers, in particular, the Hardy space and various Dirichlet spaces on bounded domains, or Sobolev spaces of holomorphic functions.

Second, we show that, again for an arbitrary reproducing kernel Hilbert space $\mathcal{H}$ contained in $\mathcal{O}(\Omega)$,

$$
\left\|\nabla^{2} \tilde{X}(z)\right\|_{z} \leq 2 \sqrt{2\left(S+n^{2}+5 n\right)}\|X\|
$$

for all $z \in \Omega$, where $S$ stands for the scalar curvature of the Riemannian metric (11). (See e.g. 19, 27] or 7] for the technical background.) Furthermore, for $n=1$ the result is optimal, and hence the left-hand side is in general unbounded if the right-hand side is: we exhibit an example of a reproducing kernel Hilbert space $\mathcal{H}$ of holomorphic functions on the unit disc $\mathbf{D}$ and an operator $X \in \mathcal{B}(\mathcal{H})$ for which $\left\|\nabla^{2} \tilde{X}(z)\right\|_{z} \rightarrow+\infty$ as $z$ tends to the boundary. Thus, in particular, the conjecture (51) does not hold for $k \geq 2$.

The proof of (8) in fact implies that

$$
S \geq-n(n+1)
$$

for any metric associated as in (1) to a reproducing kernel Hilbert space $\mathcal{H} \subset \mathcal{O}(\Omega)$. This contrasts with the fact that it is easy to devise Kähler metrics on $\Omega$ whose scalar curvature assumes arbitrarily large negative values. The inequality (9) must thus be something inherent to metrics coming from reproducing kernels. 
Finally, we give the following variant of (8) for $\nabla^{k} \tilde{X}$ with $k \geq 3$,

$$
\left\|\nabla^{k} \widetilde{X}(z)\right\|_{z}^{2} \leq r_{k}(z)\|X\|^{2},
$$

where $r_{k}$ is given by a scalar expression involving the curvature tensor $R_{i \bar{j} k \bar{l}}$ of the metric (1) and its covariant derivatives $\nabla^{m} R$ of orders $m \leq 2 k-4$.

Note that for $\mathcal{H}$ the Segal-Bargmann space on $\mathbf{C}^{n}$ or one of the usual weighted Bergman spaces on a bounded symmetric domain, $S$ is constant and $\nabla R$ vanishes; thus (8) and (10) reduce to (5), recovering the results of J. Xia and [15].

Expressions of a similar kind as our $r_{k}$ occur as the coefficients of the asymptotic expansion of the heat kernel and related geometric quantities; see e.g. [16] or [3].

The proofs of (3) and (6) appear in Section 2, the proof of (8) is contained in Section 3, along with a brief review of the required prerequisites from Kähler geometry. The proof of (10) occupies Section 4. The final section, Section 5, lists some applications and open problems.

The authors are grateful to the anonymous referee for valuable suggestions.

\section{LIPSCHITZ ESTIMATES}

The assumption $K(x, x)>0 \forall x$ implies that there exists a well-defined branch of $\log K(x, y)=: L(x, y)$ in some neighbourhood $\mathcal{U}$ of the diagonal in $\Omega \times \Omega$, with $L(x, y)$ real for $x=y$. The following assertion is standard for the ordinary Bergman kernel; its proof for a general reproducing kernel Hilbert space $\mathcal{H} \subset \mathcal{O}(\Omega)$ can be found e.g. in [14, Proposition 2.

Proposition 1. Let $z \in \Omega$. The matrix

$$
g_{j \bar{k}}(z)=\frac{\partial^{2} L(z, z)}{\partial z_{j} \partial \bar{z}_{k}}, \quad j, k=1, \ldots, n,
$$

is positive semidefinite. It is positive definite if and only if for each $v \in \mathbf{C}^{n}, v \neq 0$, there exist $f, g \in \mathcal{H}$ such that

$$
g(z) D_{v} f(z)-f(z) D_{v} g(z)=\sum_{j=1}^{n} v_{j}\left(g(z) \frac{\partial f}{\partial z_{j}}(z)-f(z) \frac{\partial g}{\partial z_{j}}(z)\right)
$$

does not vanish.

In particular, $g_{j \bar{k}}$ is positive definite on all of $\Omega$ whenever the constants and the coordinate functions belong to $\mathcal{H}$.

Let us denote by

$$
A_{z}=A(z):=\left\langle\cdot, k_{z}\right\rangle k_{z}
$$

the rank-one orthogonal projection onto $\mathbf{C} k_{z}$.

Our first result generalizes the directional derivative estimate proved for $\mathcal{H}=$ $A^{2}(\Omega)$ in [13] and also implies the Lipschitz estimates.

Theorem 2. For $T \in \mathcal{B}(\mathcal{H})$ and $v \in \mathbf{C}^{n}$,

$$
\left|D_{v} \widetilde{T}(z)\right| \leq 2\|T\|\|v\|_{z},
$$

with $\|v\|_{z}$ as in (2).

Proof. As

$$
\widetilde{T}(z)=\operatorname{tr}(T A(z)),
$$


it is standard [17] that

$$
|\widetilde{T}(x)-\widetilde{T}(y)| \leq\|T\|\left\|A_{x}-A_{y}\right\|_{\mathrm{tr}},
$$

where $\|\cdot\|_{\text {tr }}$ stands for the trace norm. A routine computation [11 shows that

$$
\left\|A_{x}-A_{y}\right\|_{\mathrm{tr}}=2 \sqrt{1-\left|\left\langle k_{x}, k_{y}\right\rangle\right|^{2}}
$$

From the Taylor series for $L(x, y)$ around $y$ we have, for $(x, y) \in \mathcal{U}$,

$$
L(x, y)=L(y, y)+\partial L(y, y) \cdot(x-y)+\frac{1}{2} \partial^{2} L(y, y) \cdot(x-y)^{2}+O\left(|x-y|^{3}\right)
$$

and similarly for $L(y, x)$, while

$$
\begin{aligned}
L(x, x)=L(y, y) & +\partial L(y, y) \cdot(x-y)+\bar{\partial} L(y, y) \cdot(\bar{x}-\bar{y}) \\
& +\frac{1}{2} \partial^{2} L(y, y) \cdot(x-y)^{2}+\frac{1}{2} \bar{\partial}^{2} L(y, y) \cdot(\bar{x}-\bar{y})^{2} \\
& +\partial \bar{\partial} L(y, y) \cdot(x-y)(\bar{x}-\bar{y})+O\left(|x-y|^{3}\right),
\end{aligned}
$$

where, for brevity, we are writing just $\partial L(y, y) \cdot(x-y)$ for $\sum_{j=1}^{n} \frac{\partial L(y, y)}{\partial y_{j}}\left(x_{j}-y_{j}\right)$ and so on. Subtracting, we get

$$
\begin{aligned}
\log \left|\left\langle k_{x}, k_{y}\right\rangle\right|^{2} & =L(x, y)+L(y, x)-L(x, x)-L(y, y) \\
& =-\partial \bar{\partial} L(y, y) \cdot(x-y)(\bar{x}-\bar{y})+O\left(|x-y|^{3}\right) .
\end{aligned}
$$

It follows that

$$
\begin{aligned}
1-\left|\left\langle k_{x}, k_{y}\right\rangle\right|^{2} & =1-e^{\log \left|\left\langle k_{x}, k_{y}\right\rangle\right|^{2}} \\
& =\partial \bar{\partial} L(y, y) \cdot(x-y)(\bar{x}-\bar{y})+O\left(|x-y|^{3}\right) \\
& =\sum_{j, k} g_{j \bar{k}}(y)\left(x_{j}-y_{j}\right)\left(\bar{x}_{k}-\bar{y}_{k}\right)+O\left(|x-y|^{3}\right),
\end{aligned}
$$

by (11).

It was shown in [15] that the function $A(z)$ has derivatives of all orders in the trace-norm topology, and thus

$$
D_{v} \widetilde{T}(z)=\operatorname{tr}\left(T D_{v} A(z)\right)
$$

exists for all $z \in \Omega$ and $v \in \mathbf{C}^{n}$. On the other hand, from (12), (13) and (14) we have for any real $t \neq 0$ sufficiently small,

$$
\begin{aligned}
|\widetilde{T}(z+t v)-\widetilde{T}(z)| & \leq 2\|T\| \sqrt{1-\left|\left\langle k_{z+t v}, k_{z}\right\rangle\right|^{2}} \\
& \leq 2\|T\| \sqrt{t^{2}\|v\|_{z}^{2}+O\left(t^{3}\right)}
\end{aligned}
$$

that is,

$$
\left|\frac{\widetilde{T}(z+t v)-\widetilde{T}(z)}{t}\right| \leq 2\|T\| \sqrt{\|v\|_{z}^{2}+O(t)} .
$$

Letting $t \rightarrow 0$, the assertion follows.

Corollary 3. For $T \in \mathcal{B}(\mathcal{H})$ and $x, y \in \Omega$,

$$
|\widetilde{T}(x)-\widetilde{T}(y)| \leq 2\|T\| \beta(x, y) .
$$

Indeed, this is immediate from the standard definition of $\beta(x, y)$ as the infimum of lengths, with respect to (2), of curves joining $x$ to $y$. The next corollary is even more straightforward. 
Corollary 4. If $\|v\|_{z}$ is majorized by a multiple of the Euclidean length $|v|$, then $\widetilde{T}$ is Lipschitz.

From the formula (22) in $\S 18.1$ of [2], one learns that the Mittag-Leffler function

$$
E_{\alpha, \alpha}(t)=\sum_{k=0}^{\infty} \frac{t^{k}}{\Gamma(k \alpha+\alpha)}
$$

has the asymptotic expansion

$$
E_{\alpha, \alpha}(t)=e^{t^{1 / \alpha}}+O\left(e^{-\theta|t|^{1 / \alpha}}\right)
$$

as $t \rightarrow \infty$ in a small conical neighbourhood of the positive real axis, with some $\theta>0$. By Cauchy's formula (integrating over circles of radius 1 around $t$ ), it also follows that

$$
E_{\alpha, \alpha}^{(k)}(t)=\left(e^{t^{1 / \alpha}}\right)^{(k)}+O\left(e^{-\theta|t|^{1 / \alpha}}\right),
$$

for any $k=1,2,3, \ldots$ Since $E_{\alpha, \alpha}^{(k)}>0$ on the positive real axis, there exists a branch $G(t)$ of $\log E^{(n-1)}(t)$ in some neighbourhood of $\mathbf{R}^{+}$, and (16) gives

$$
G^{(k)}(t)=O\left(t^{(k / \alpha)-k}\right)
$$

as $t \rightarrow+\infty$ through $\mathbf{R}^{+}$, for $k=1,2$. Recalling now the formula [10]

$$
K(x, y)=E_{2 / m, 2 / m}^{(n-1)}(x \cdot \bar{y}) \equiv F(x \cdot \bar{y})
$$

for the reproducing kernel of $A^{2}\left(\mathbf{C}^{n}, d \mu_{m}\right)$ with $\mu_{m}$ given by (7), and using the relation

$$
\begin{aligned}
g_{j \bar{k}}(z) & =\frac{\delta_{j k} F^{\prime}\left(|z|^{2}\right)}{F\left(|z|^{2}\right)}+\bar{z}_{j} z_{k} \frac{F\left(|z|^{2}\right) F^{\prime \prime}\left(|z|^{2}\right)-\left|F^{\prime}\left(|z|^{2}\right)\right|^{2}}{F\left(|z|^{2}\right)^{2}} \\
& =\delta_{j k} G^{\prime}\left(|z|^{2}\right)+\bar{z}_{j} z_{k} G^{\prime \prime}\left(|z|^{2}\right),
\end{aligned}
$$

it follows that

$$
\|v\|_{z}^{2}=\|v\|^{2} G^{\prime}\left(|z|^{2}\right)+|\langle v, z\rangle|^{2} G^{\prime \prime}\left(|z|^{2}\right)=O\left(|z|^{2 m-2}|v|^{2}\right) .
$$

In particular, for $m \leq 1$ the last corollary applies, and thus $\widetilde{T}$ is globally Lipschitz. This is Corollary 4 of $\left[8\right.$. Of course, for $m=2$ (i.e. $\alpha=1$ ), we get $G^{\prime \prime}=0$ and $\|v\|_{z}=|v|$, recovering thus from our last corollary the original result of [11, too.

As in 12 and [13, we can also show that the estimates in Theorem 2 and Corollary 3 are sharp. The only missing piece is the analogue of the formula (4) in [13, which was proved for $\mathcal{H}=A^{2}(\Omega)$ in 24]; for general $\mathcal{H}$, we provide it as the next proposition.

Proposition 5. For $v \in \mathbf{C}^{n}$, the directional derivative of the function $A(z)=$ $\left\langle\cdot, k_{z}\right\rangle k_{z}$ satisfies

$$
\left\|D_{v} A(z)\right\|_{\mathrm{tr}}=2\|v\|_{z} .
$$

Proof. From (13) and (14) we get, for any $t \neq 0$,

$$
\left\|\frac{A_{z+t v}-A_{z}}{t}\right\|_{\mathrm{tr}}=\frac{2 \sqrt{t^{2}\|v\|_{z}^{2}+O\left(t^{3}\right)}}{|t|}=2 \sqrt{\|v\|_{z}^{2}+O(t)} .
$$

Letting $t \rightarrow 0$ yields the claim. 
Theorem 6. For any $z \in \Omega$ and $v \in \mathbf{C}^{n}$, the operator $X=D_{v} A(z)$ satisfies

$$
\left|D_{v} \widetilde{X}(z)\right|=2\|X\|\|v\|_{z} .
$$

Consequently, the constant 2 in (11) and (15) is optimal.

Proof. The proof is the same as the proof of Theorem 3.3 in 13, with (4) from that paper substituted by the preceding proposition.

\section{SECOND-ORDER COVARIANT DERIVATIVES}

From now on we assume that the condition in Proposition 1 is fulfilled, so that the metric (10) is Riemannian and not just semi-Riemannian. Let $g^{\bar{k} j}(z)$ denote the inverse matrix to $g_{j \bar{k}}(z)$, i.e.

$$
g_{j \bar{k}} g^{\bar{k} l}=g^{\bar{l} k} g_{k \bar{j}}= \begin{cases}0 & \text { if } l \neq j \\ 1 & \text { if } l=j,\end{cases}
$$

where we have started using the usual convention of summing automatically over any index which occurs once in the upper and once in the lower position. For any covariant $k$-tensor field $T_{\alpha_{1} \ldots \alpha_{k}}$ on $\Omega$, its covariant derivative is the covariant $(k+1)$ tensor field $\nabla T$ defined as

$$
(\nabla T)_{\alpha_{1} \ldots \alpha_{k} \beta}=D_{\beta} T_{\alpha_{1} \ldots \alpha_{k}}-\sum_{m=1}^{k} \Gamma_{\beta \alpha_{m}}^{\gamma} T_{\alpha_{1} \ldots \alpha_{m-1} \gamma \alpha_{m+1} \ldots \alpha_{k}} .
$$

The notation $T_{\alpha_{1} \ldots \alpha_{k} / \beta}$ is also sometimes used instead of $(\nabla T)_{\alpha_{1} \ldots \alpha_{k} \beta}$. Here $D_{\beta}$ denotes the directional derivative in the direction of the $\beta$-th coordinate, and the Greek letters $\alpha_{1}, \ldots, \alpha_{k}, \beta, \gamma$ can be either the unbarred indices such as $i, j, k, \ldots$ or the barred ones $\bar{j}, \bar{k}, \bar{l}, \ldots$. The Riemann-Christoffel coefficients $\Gamma_{\beta \alpha}^{\gamma}$ are given by

$$
\Gamma_{j k}^{l}=g^{\bar{m} l} g_{j k \bar{m}}, \quad \Gamma_{\overline{j k}}^{\bar{l}}=g^{\bar{l} m} g_{m \bar{j} \bar{k}}, \quad \Gamma_{\bar{j} k}^{l}=\Gamma_{\overline{j k}}^{l}=\Gamma_{j \bar{k}}^{l}=\Gamma_{\bar{j} k}^{\bar{l}}=\Gamma_{j k}^{\bar{l}}=\Gamma_{j \bar{k}}^{\bar{l}}=0,
$$

where

$$
g_{j k \bar{m}}=\frac{\partial^{3} L(z, z)}{\partial z_{j} \partial z_{k} \partial \bar{z}_{m}}=\frac{\partial g_{k \bar{m}}}{\partial z_{j}}=\frac{\partial g_{j \bar{m}}}{\partial z_{k}}
$$

and similarly for $g_{m \overline{j k}}, g_{\bar{j} k \bar{l} m}$, and so forth.

It will be convenient to split $\nabla T$ into its holomorphic and anti-holomorphic parts

$$
\begin{aligned}
& (\partial T)_{\alpha_{1} \ldots \alpha_{k} j}=\partial_{j} T_{\alpha_{1} \ldots \alpha_{k}}-\sum_{m=1}^{k} \Gamma_{j \alpha_{m}}^{l} T_{\alpha_{1} \ldots \alpha_{m-1} l \alpha_{m+1} \ldots \alpha_{k}}, \\
& (\bar{\partial} T)_{\alpha_{1} \ldots \alpha_{k} \bar{j}}=\bar{\partial}_{j} T_{\alpha_{1} \ldots \alpha_{k}}-\sum_{m=1}^{k} \Gamma_{\bar{j} \alpha_{m}}^{\bar{l}} T_{\alpha_{1} \ldots \alpha_{m-1} \bar{l} \alpha_{m+1} \ldots \alpha_{k}},
\end{aligned}
$$

where we also introduced the shorthand $\partial_{j}=\partial / \partial z_{j}, \bar{\partial}_{j}=\partial / \partial \bar{z}_{j}$.

Since $\nabla T$ is again a covariant tensor field, the procedure can be iterated, yielding higher-order covariant derivatives $\nabla^{m} T, m=2,3, \ldots$, which can again be split into the various holomorphic and anti-holomorphic components $\partial^{m_{1}} \bar{\partial}^{m_{2}} \partial^{m_{3}} \ldots T$, $m_{1}+m_{2}+m_{3}+\cdots=m$. In particular, for $f$ a function on $\Omega$, we have

$$
\begin{aligned}
& f_{/ j}=\partial_{j} f, \quad f_{/ \bar{k}}=\bar{\partial}_{k} f, \quad f_{/ j \bar{k}}=f_{/ \bar{k} j}=\partial_{j} \bar{\partial}_{k} f, \\
& f_{/ j l}=\partial_{l} \partial_{j} f-\Gamma_{l j}^{m} \partial_{m} f=\partial_{l} \partial_{j} f-g^{\bar{k} m} g_{l j \bar{k}} \partial_{m} f .
\end{aligned}
$$


The curvature tensor $R_{i \bar{j} k \bar{l}}$ of the metric (1) is given by

$$
R_{i \bar{j} k \bar{l}}=g_{i \bar{j} k \bar{l}}-g^{\bar{q} p} g_{i k \bar{q}} g_{\bar{j} \bar{l} p} .
$$

The contraction

$$
g^{\bar{l} i} g^{\bar{j} k} R_{i \bar{j} k \bar{l}}=S
$$

is the scalar curvature.

Recall that, quite generally, the norm of a tensor $T_{\alpha_{1} \alpha_{2} \ldots}$ is given by

$$
\left\|T_{\alpha_{1} \alpha_{2} \ldots}\right\|^{2}=g^{\bar{\beta}_{1} \alpha_{1}} g^{\bar{\beta}_{2} \alpha_{2}} \ldots T_{\alpha_{1} \alpha_{2} \ldots} \overline{T_{\beta_{1} \beta_{2} \ldots}} .
$$

Accordingly, we set, for a function $f$ on $\Omega$,

$$
\|\partial f\|^{2}=g^{\bar{k} j} f_{/ j} \overline{f_{/ k}}, \quad\|\bar{\partial} f\|^{2}=g^{\bar{k} j} f_{/ \bar{k}} \overline{f_{/ \bar{j}}},
$$

and 2

$$
\|\nabla f\|^{2}:=2\left(\|\partial f\|^{2}+\|\bar{\partial} f\|^{2}\right) .
$$

Of course, all these quantities depend on the point $z$ where they are taken, so strictly speaking we should write $\|\partial f(z)\|_{z}$, and so on.

Note that

$$
\|\partial f(z)\|_{z}=\sup \left\{\left|v^{j} f_{/ j}(z)\right|:\|v\|_{z} \leq 1\right\}
$$

and similarly for $\|\bar{\partial} f\|$. Indeed, denoting for a second by $\gamma$ the $n \times n$ (positive definite) matrix $g_{k \bar{j}}$, and identifying $v^{j}$ with the column $n$-vector in $\mathbf{C}^{n}$, we have $\|v\|_{z}^{2}=\langle\gamma \bar{v}, \bar{v}\rangle$, and

$$
\begin{aligned}
\sup _{\langle\bar{v}, \bar{v}\rangle \leq 1}|\langle\partial f, \bar{v}\rangle|^{2} & =\sup _{|w| \leq 1}\left|\left\langle\partial f, \gamma^{-1 / 2} w\right\rangle\right|^{2} \\
& =\sup _{|w| \leq 1}\left|\left\langle\gamma^{-1 / 2} \partial f, w\right\rangle\right|^{2} \\
& =\left|\gamma^{-1 / 2} \partial f\right|^{2}=\left\langle\gamma^{-1} \partial f, \partial f\right\rangle,
\end{aligned}
$$

proving the claim. Similarly,

$$
\|\partial \partial f\|^{2}=g^{\bar{k} j} g^{\bar{q} p} f_{/ j p} \overline{f_{/ k q}}=\sup \left\{\left|v^{j p} f_{/ j p}\right|: g_{j_{1} \bar{j}_{2}} g_{p_{1} \bar{p}_{2}} v^{j_{1} p_{1}} \overline{v^{j_{2} p_{2}}} \leq 1\right\} .
$$

The last expression is a summand in

$$
\left\|\nabla^{2} f\right\|^{2}=4\left(\|\partial \partial f\|^{2}+\|\overline{\partial \partial} f\|^{2}+\|\partial \bar{\partial} f\|^{2}+\|\bar{\partial} \partial f\|^{2}\right) .
$$

For some background on Kähler geometry, the reader is referred e.g. to [22, Chapter IX, or [7, Chapter VIII.

We begin with a coordinate-free version of the estimate (11) from the last section.

Theorem 7. For any $T \in \mathcal{B}(\mathcal{H})$ and $z \in \Omega$,

$$
\|\partial \widetilde{T}(z)\|_{z} \leq\|T\|, \quad\|\bar{\partial} \widetilde{T}(z)\|_{z} \leq\|T\| .
$$

\footnotetext{
${ }^{2}$ The factor "2" is inserted to conform with the usual definition $\|\nabla f\|^{2}=\left|\frac{\partial f}{\partial x}\right|^{2}+\left|\frac{\partial f}{\partial y}\right|^{2}$ of the norm of the gradient of a function of $z=x+i y \in \mathbf{C}$. Similarly, for $\nabla^{m}$ we get a factor of $2^{m}$.
} 
Proof. Since $\widetilde{T^{*}}=\overline{\widetilde{T}}$, hence $\widetilde{T}_{/ \bar{j}}=\overline{\widetilde{T}^{*} / j}$, while $\left\|T^{*}\right\|=\|T\|$, it is enough to prove the estimate for $\|\partial \widetilde{T}\|$. From $\widetilde{T}(z)=\operatorname{tr}(T A(z))$ we get

$$
\widetilde{T}_{/ j}(z)=\operatorname{tr}\left(T A_{/ j}(z)\right)
$$

where

$$
\begin{aligned}
A_{/ j}(z) & =\partial_{j} A(z)=\partial_{j}\left(K^{-1}\left\langle\cdot, K_{z}\right\rangle K_{z}\right) \\
& =-\frac{\partial_{j} K}{K^{2}}\left\langle\cdot, K_{z}\right\rangle K_{z}+\frac{1}{K}\left\langle\cdot, \bar{\partial}_{j, z} K_{z}\right\rangle K_{z},
\end{aligned}
$$

since $K_{z}$ depends anti-holomorphically on $z$; here we started omitting, for the sake of brevity, the arguments $(z, z)$ and $z$ in $K(z, z), \partial_{j} K(z, z), A(z)$, and so on. Thus

$$
A_{/ j}=\left\langle\cdot, a_{j}\right\rangle K_{z}, \quad \text { where } a_{j}=-\frac{\bar{\partial}_{j} K}{K^{2}} K_{z}+\frac{1}{K} \bar{\partial}_{j, z} K_{z} .
$$

It follows that for any $v=\left(v^{j}\right) \in \mathbf{C}^{n}$,

$$
\begin{aligned}
\left|v^{j} \widetilde{T}_{/ j}\right|^{2} & =\left|\operatorname{tr}\left(T v^{j} A_{/ j}\right)\right|^{2}=\left|\left\langle T K_{z}, \bar{v}^{j} a_{j}\right\rangle\right|^{2} \\
& \leq\|T\|^{2} \cdot K \cdot \bar{v}^{j} v^{k}\left\langle a_{j}, a_{k}\right\rangle .
\end{aligned}
$$

Now

$$
\begin{aligned}
\left\langle a_{j}, a_{k}\right\rangle= & \frac{\bar{\partial}_{j} K}{K^{2}} \frac{\partial_{k} K}{K^{2}}\left\langle K_{z}, K_{z}\right\rangle-\frac{\bar{\partial}_{j} K}{K^{3}}\left\langle K_{z}, \bar{\partial}_{k, z} K_{z}\right\rangle \\
& \quad-\frac{\partial_{k} K}{K^{3}}\left\langle\bar{\partial}_{j, z} K_{z}, K_{z}\right\rangle+\frac{1}{K^{2}}\left\langle\bar{\partial}_{j, z} K_{z}, \bar{\partial}_{k, z} K_{z}\right\rangle \\
= & \frac{\bar{\partial}_{j} K \partial_{k} K}{K^{3}}-\frac{\bar{\partial}_{j} K}{K^{3}} \partial_{k} K-\frac{\partial_{k} K}{K^{3}} \bar{\partial}_{j} K+\frac{1}{K^{2}} \partial_{k} \bar{\partial}_{j} K \\
= & -\frac{\bar{\partial}_{j} K \partial_{k} K}{K^{3}}+\frac{\bar{\partial}_{j} \partial_{k} K}{K^{2}} \\
= & \frac{1}{K}\left[-L_{\bar{j}} L_{k}+\left(L_{\bar{j}} L_{k}+L_{\bar{j} k}\right)\right]=\frac{L_{\bar{j} k}}{K},
\end{aligned}
$$

where we have introduced one more piece of shorthand by setting

$$
L_{\bar{j}}=\bar{\partial}_{j} L=\frac{\bar{\partial}_{j} K}{K}, \quad L_{k}=\partial_{k} L, \quad L_{\bar{j} k}=\bar{\partial}_{j} \partial_{k} L, \quad \text { etc. },
$$

and have used the formula

$$
\frac{\bar{\partial}_{j} \partial_{k} K}{K}=e^{-L} \bar{\partial}_{j} \partial_{k} e^{L}=L_{\bar{j}} L_{k}+L_{\bar{j} k}
$$

We thus get

$$
\left|v^{j} \widetilde{T}_{/ j}\right|^{2} \leq\|T\|^{2} L_{\bar{j} k} \bar{v}^{j} v^{k}=\|T\|^{2}\|v\|_{z}^{2},
$$

since $L_{\bar{j} k}=g_{k \bar{j}}$. By (20) also follows that

$$
\|\partial \widetilde{T}\|^{2} \leq\|T\|^{2},
$$

completing the proof of the theorem.

The rest of this section is devoted to estimating $\left\|\nabla^{2} \widetilde{T}\right\|$. 
Theorem 8. For $T \in \mathcal{B}(\mathcal{H})$ and $z \in \Omega$,

$$
\|\partial \partial \widetilde{T}(z)\|_{z} \leq \sqrt{S+n^{2}+n}\|T\|,
$$

where $S$ is the scalar curvature (19). A similar result holds for $\|\overline{\partial \partial} \widetilde{T}(z)\|_{z}$.

Proof. Again, passing from $T$ to $T^{*}$, it is enough to prove the assertion concerning $\partial \partial \widetilde{T}$. As before, we have

$$
\widetilde{T}_{/ j m}(z)=\operatorname{tr}\left(T A_{/ j m}(z)\right),
$$

where (we are again omitting the arguments $z$ or $(z, z)$ )

$$
\begin{aligned}
A_{/ j m} & =\partial_{m} A_{/ j}-\Gamma_{m j}^{p} A_{/ p} \\
& =\left\langle\cdot, \bar{\partial}_{m, z} a_{j}\right\rangle K_{z}-\Gamma_{m j}^{p}\left\langle\cdot, a_{p}\right\rangle K_{z} \\
& =\left\langle\cdot, \bar{\partial}_{m, z} a_{j}-\overline{\Gamma_{m j}^{p}} a_{p}\right\rangle K_{z},
\end{aligned}
$$

since $\partial_{m, z} K_{z}=0$. Thus for any $\mathbf{v}=\left(v^{j m}\right) \in \mathbf{C}^{n^{2}}$,

$$
\begin{aligned}
\left|v^{j m} \widetilde{T}_{/ j m}\right|^{2} & =\left|\operatorname{tr}\left(T v^{j m} A_{/ j m}\right)\right|^{2} \\
& \leq\|T\|^{2} K\left\|\bar{v}^{j m}\left(\bar{\partial}_{m, z} a_{j}-\overline{\Gamma_{m j}^{p}} a_{p}\right)\right\|^{2} .
\end{aligned}
$$

To compute the last norm-square, note that by (23),

$$
\left\langle\overline{\Gamma_{m j}^{p}} a_{p}, \overline{\Gamma_{k l}^{q}} a_{q}\right\rangle=\Gamma_{k l}^{q} \overline{\Gamma_{m j}^{p}}\left\langle a_{p}, a_{q}\right\rangle=g^{\bar{r} q} g_{k l \bar{r}} g^{\bar{p} s} g_{s \bar{m} \bar{j}} \frac{g_{\bar{p} q}}{K}=\frac{g^{\bar{r} q} g_{k l \bar{r}} g_{q \bar{m} \bar{j}}}{K} .
$$

Next,

$$
\begin{aligned}
\left\langle\bar{\partial}_{m, z} a_{j}, a_{q}\right\rangle= & \left\langle\bar{\partial}_{m, z}\left(-\frac{\bar{\partial}_{j} L}{K} K_{z}+\frac{1}{K} \bar{\partial}_{j, z} K_{z}\right),-\frac{\bar{\partial}_{q} L}{K} K_{z}+\frac{1}{K} \bar{\partial}_{q, z} K_{z}\right\rangle \\
= & \left\langle\left(-\frac{\bar{\partial}_{m} \bar{\partial}_{j} L}{K}+\frac{\bar{\partial}_{j} L \bar{\partial}_{m} L}{K}\right) K_{z}-\frac{\bar{\partial}_{j} L}{K} \bar{\partial}_{m, z} K_{z}-\frac{\bar{\partial}_{m} L}{K} \bar{\partial}_{j, z} K_{z}\right. \\
& \left.\quad+\frac{1}{K} \bar{\partial}_{m, z} \bar{\partial}_{j, z} K_{z},-\frac{\bar{\partial}_{q} L}{K} K_{z}+\frac{1}{K} \bar{\partial}_{q, z} K_{z}\right\rangle \\
= & \left(-\frac{L_{\bar{m} \bar{j}}}{K}+\frac{L_{\bar{j}} L_{\bar{m}}}{K}\right) \cdot(\underbrace{-\frac{L_{q}}{K} K+\frac{1}{K} \partial_{q} K}_{0}) \\
& \quad-\frac{L_{\bar{j}}}{K}\left(-\frac{L_{q}}{K} \bar{\partial}_{m} K+\frac{1}{K} \bar{\partial}_{m} \partial_{q} K\right) \\
& \quad-\frac{L_{\bar{m}}}{K}\left(-\frac{L_{q}}{K} \bar{\partial}_{j} K+\frac{1}{K} \bar{\partial}_{j} \partial_{q} K\right) \\
& +\frac{1}{K}\left(-\frac{L_{q}}{K} \bar{\partial}_{m} \bar{\partial}_{j} K+\frac{1}{K} \partial_{q} \bar{\partial}_{m} \bar{\partial}_{j} K\right) \\
= & -\frac{L_{\bar{j}}}{K} L_{\bar{m} q}-\frac{L_{\bar{m}}}{K} L_{\bar{j} q}+\frac{1}{K}\left[-L_{q}\left(L_{\bar{m}} L_{\bar{j}}+L_{\bar{m} \bar{j}}\right)\right. \\
& \left.+\left(L_{q} L_{\bar{m}} L_{\bar{j}}+L_{q} L_{\bar{m} \bar{j}}+L_{\bar{m}} L_{q \bar{j}}+L_{\bar{j}} L_{q \bar{m}}+L_{q \bar{m} \bar{j}}\right)\right] \\
= & \frac{1}{K} L_{q \bar{m} \bar{j}}=\frac{1}{K} g_{q \bar{m} \bar{j}} .
\end{aligned}
$$

Thus

$$
\left\langle\bar{\partial}_{m, z} a_{j}, \overline{\Gamma_{k l}^{q}} a_{q}\right\rangle=g^{\bar{p} q} g_{\bar{p} k l} \cdot \frac{1}{K} g_{q \bar{m} \bar{j}} .
$$


Similarly,

$$
\left\langle\overline{\Gamma_{m j}^{p}} a_{p}, \bar{\partial}_{k, z} a_{l}\right\rangle=g^{\bar{p} s} g_{s \bar{m} \bar{j}} \frac{1}{K} g_{\bar{p} k l} .
$$

Finally, one more computation as above yields

$$
\left\langle\bar{\partial}_{m, z} a_{j}, \bar{\partial}_{k, z} a_{l}\right\rangle=\frac{1}{K}\left(L_{k l \bar{m} \bar{j}}+L_{k \bar{m}} L_{l \bar{j}}+L_{k \bar{j}} L_{l \bar{m}}\right) .
$$

Putting all four pieces together, we therefore obtain

$$
\begin{aligned}
\left\|\bar{v}^{j m}\left(\bar{\partial}_{m, z} a_{j}-\overline{\Gamma_{m j}^{p}} a_{p}\right)\right\|^{2} & =\bar{v}^{j m} v^{k l}\left\langle\bar{\partial}_{m, z} a_{j}-\overline{\Gamma_{m j}^{p}} a_{p}, \bar{\partial}_{k, z} a_{l}-\overline{\Gamma_{k l}^{q}} a_{q}\right\rangle \\
& =\frac{\bar{v}^{j m} v^{k l}}{K}\left(L_{k l \bar{m} \bar{j}}+L_{k \bar{m}} L_{l \bar{j}}+L_{k \bar{j}} L_{l \bar{m}}-g^{\bar{p} q} g_{k l \bar{p}} g_{\bar{m} \bar{j} q}\right) \\
& =\frac{\bar{v}^{j m} v^{k l}}{K}\left(R_{k \bar{j} l \bar{m}}+g_{k \bar{m}} g_{l \bar{j}}+g_{k \bar{j}} g_{l \bar{m}}\right) .
\end{aligned}
$$

Denote, for a second, $\mathbf{Q}_{\bar{j} \bar{m}, k l}:=R_{k \bar{j} l \bar{m}}+g_{k \bar{m}} g_{l \bar{j}}+g_{k \bar{j}} g_{l \bar{m}}$, and let $\langle\langle\cdot, \cdot\rangle\rangle$ stand for the scalar product in $\mathbf{C}^{n^{2}}$. Thus we have shown that

$$
K \cdot\left\|\bar{v}^{j m}\left(\bar{\partial}_{m, z} a_{j}-\overline{\Gamma_{m j}^{p}} a_{p}\right)\right\|^{2}=\langle\langle\mathbf{Q v}, \mathbf{v}\rangle\rangle .
$$

Let $\mathbf{g}$ stand for the $n^{2} \times n^{2}$ matrix $\mathbf{g}_{\bar{j} \bar{m}, k l}=g_{k \bar{j}} g_{l \bar{m}}$. Then we have

$$
\begin{aligned}
\sup \left\{\bar{v}^{j m} v^{k l} \mathbf{Q}_{\bar{j} \bar{m}, k l}: g_{k \bar{j}} g_{l \bar{m}} v^{k l} \bar{v}^{j m} \leq 1\right\} & =\sup \{\langle\mathbf{Q v}, \mathbf{v}\rangle\rangle:\langle\langle\mathbf{g v}, \mathbf{v}\rangle\rangle \leq 1\} \\
& \left.=\sup \left\{\left\langle\mathbf{Q g}^{-1 / 2} \mathbf{w}, \mathbf{g}^{-1 / 2} \mathbf{w}\right\rangle\right\rangle:\langle\langle\mathbf{w}, \mathbf{w}\rangle\rangle \leq 1\right\} \\
& \left.=\sup \left\{\left\langle\mathbf{g}^{-1 / 2} \mathbf{Q g}^{-1 / 2} \mathbf{w}, \mathbf{w}\right\rangle\right\rangle:\langle\langle\mathbf{w}, \mathbf{w}\rangle\rangle \leq 1\right\} .
\end{aligned}
$$

Since the trace of an operator $\mathbf{X} \in \mathcal{B}\left(\mathbf{C}^{n^{2}}\right)$ is equal to the sum of $\langle\langle\mathbf{X} \mathbf{w}, \mathbf{w}\rangle\rangle$ over $\mathbf{w}$ in an (arbitrary) orthonormal basis of $\mathbf{C}^{n^{2}}$, we can continue with

$$
\begin{aligned}
& \leq \operatorname{tr}\left(\mathbf{g}^{-1 / 2} \mathbf{Q g}^{-1 / 2}\right)=\operatorname{tr}\left(\mathbf{g}^{-1} \mathbf{Q}\right) \\
& =g^{\bar{j} k} g^{\bar{m} l} \mathbf{Q}_{\bar{j} \bar{m}, k l} \\
& =g^{\bar{j} k} g^{\bar{m} l}\left(R_{k \bar{j} l \bar{m}}+g_{k \bar{m}} g_{l \bar{j}}+g_{k \bar{j}} g_{l \bar{m}}\right) \\
& =g^{\bar{j} k}\left(R_{k \bar{j} l}{ }^{l}+g_{k \bar{j}}+n g_{k \bar{j}}\right) \\
& =S+n+n^{2} .
\end{aligned}
$$

Hence by (21),

$$
\begin{aligned}
\|\partial \partial \widetilde{T}\|^{2} & =\sup \left\{\left|v^{j m} \widetilde{T}_{/ j m}\right|^{2}:\langle\langle\mathbf{g v}, \mathbf{v}\rangle\rangle \leq 1\right\} \\
& \leq\|T\|^{2} \sup \{\langle\langle\mathbf{Q v}, \mathbf{v}\rangle\rangle:\langle\langle\mathbf{g v}, \mathbf{v}\rangle\rangle \leq 1\} \\
& \leq\left(S+n^{2}+n\right)\|T\|^{2},
\end{aligned}
$$

completing the proof.

We also have the analogous estimate for the mixed covariant derivatives.

Theorem 9. For $T \in \mathcal{B}(\mathcal{H})$ and $z \in \Omega$,

$$
\|\bar{\partial} \partial \widetilde{T}(z)\|_{z}=\|\partial \bar{\partial} \widetilde{T}(z)\|_{z} \leq 2 \sqrt{n}\|T\| .
$$


Proof. The assertion for $\partial \bar{\partial} \widetilde{T}$ again follows from the one for $\bar{\partial} \partial \widetilde{T}$ by passing from $T$ to $T^{*}$ and making complex conjugation. Thus we only need to estimate

$$
\|\bar{\partial} \partial \widetilde{T}\|^{2}=g^{\bar{l} j} g^{\bar{k} m} \widetilde{T}_{/ j \bar{k}} \widetilde{T}_{/ l \bar{m}}
$$

Since the argument is completely similar to the preceding two proofs, we will be brief. One has

$$
\begin{aligned}
A_{/ j \bar{k}} & =\partial_{j} \bar{\partial}_{k} A=\bar{\partial}_{k}\left(\left\langle\cdot, a_{j}\right\rangle K_{z}\right)=\left\langle\cdot, \partial_{k} a_{j}\right\rangle K_{z}+\left\langle\cdot, a_{j}\right\rangle \bar{\partial}_{k, z} K_{z} \\
& =\left\langle\cdot,\left(-\frac{L_{\bar{j} k}}{K}+\frac{L_{\bar{j}} L_{k}}{K}\right) K_{z}-\frac{L_{k}}{K} \bar{\partial}_{j, z} K_{z}\right\rangle K_{z}+\left\langle\cdot, a_{j}\right\rangle \bar{\partial}_{k, z} K_{z} \\
& =K\left\langle\cdot, a_{j}\right\rangle a_{k}-\frac{L_{j \bar{k}}}{K}\left\langle\cdot, K_{z}\right\rangle K_{z} .
\end{aligned}
$$

Thus, for any $v=\left(v^{j}\right), w=\left(w^{k}\right)$ in $\mathbf{C}^{n}$,

$$
\begin{aligned}
\left|v^{j} \bar{w}^{k} \widetilde{T}_{/ j \bar{k}}\right| & =\left|\operatorname{tr}\left(T v^{j} \bar{w}^{k} A_{/ j \bar{k}}\right)\right| \\
& \leq\|T\|\left\|K\left\langle\cdot, \bar{v}^{j} a_{j}\right\rangle \bar{w}^{k} a_{k}-\frac{v^{j} \bar{w}^{k} L_{j \bar{k}}}{K}\left\langle\cdot, K_{z}\right\rangle K_{z}\right\|_{\mathrm{tr}} \\
& \leq\|T\|\left[K\left\|\bar{v}^{j} a_{j}\right\|\left\|\bar{w}^{k} a_{k}\right\|+\|v\|_{z}\|w\|_{z}\right] \\
& =2\|T\|\|v\|_{z}\|w\|_{z},
\end{aligned}
$$

since $\left\|\bar{v}^{j} a_{j}\right\|^{2}=\|v\|_{z}^{2} / K$ by (23), and similarly for $w^{k}$.

Identifying, for a moment, $\left(\widetilde{T}_{/ j \bar{k}}\right) \equiv F$ with an $n \times n$ matrix operating on $\mathbf{C}^{n}$, and letting $\gamma$ again stand for the matrix $g_{j \bar{k}}$, we have

$$
\begin{aligned}
g^{\overline{l j}} g^{\bar{k} m} \widetilde{T}_{/ j \bar{k}} \widetilde{T}_{/ l \bar{m}} & =\operatorname{tr}\left(\boldsymbol{\gamma}^{-1} F \gamma^{-1} F^{*}\right) \\
& =\operatorname{tr}\left(\boldsymbol{\gamma}^{-1 / 2} F \gamma^{-1} F^{*} \gamma^{-1 / 2}\right) \\
& \leq n \sup _{|e|=1}\left\langle\gamma^{-1 / 2} F \gamma^{-1} F^{*} \gamma^{-1 / 2} e, e\right\rangle \\
& =n \sup _{|e|=1}\left|\gamma^{-1 / 2} F^{*} \gamma^{-1 / 2} e\right|^{2} \\
& =n \sup _{|e|=|f|=1}\left|\left\langle F^{*} \gamma^{-1 / 2} e, \gamma^{-1 / 2} f\right\rangle\right|^{2} \\
& =n \sup _{\left|\gamma^{1 / 2} \bar{v}\right|=\left|\boldsymbol{\gamma}^{1 / 2} \bar{w}\right|=1}\left|\left\langle F^{*} \bar{v}, \bar{w}\right\rangle\right|^{2} \\
& =n \sup _{\langle\gamma \bar{v}, \bar{v}\rangle=\langle\gamma \bar{w}, \bar{w}\rangle=1}|\langle F \bar{w}, \bar{v}\rangle|^{2} \\
& =n \sup _{\|v\|_{z}=\|w\|_{z}=1}\left|v^{j} \bar{w}^{k} \widetilde{T}_{/ j \bar{k}}\right|^{2} \\
& \leq 4 n\|T\|^{2},
\end{aligned}
$$

by (27), proving the claim.

Corollary 10. For $T \in \mathcal{B}(\mathcal{H})$ and $z \in \Omega$,

$$
\left\|\nabla^{2} \widetilde{T}(z)\right\|_{z} \leq 2 \sqrt{2\left(S+n^{2}+5 n\right)}\|T\| .
$$

As noted in the introduction, it is also a consequence of Theorem 8 that

$$
S \geq-n(n+1) \text {. }
$$


It is easy to see that this need not be true for general Kähler metrics: for instance, the metric on $\mathbf{C}$ given by the potential $\frac{2}{\rho} \log \left(1+|z|^{2}\right)$, with arbitrary $\rho>0$, that is,

$$
g_{1 \overline{1}}=\partial_{1} \bar{\partial}_{1} \log \left(1+|z|^{2}\right)^{2 / \rho}=\frac{2}{\rho\left(1+|z|^{2}\right)^{2}},
$$

has constant scalar curvature $S=-\rho$.

We also note that the estimate in Theorem 8 cannot be improved in general: in particular, $\|\partial \partial \widetilde{T}(z)\|_{z}^{2}$ need not be bounded on $\Omega$ if $S$ is not.

Example 11. Let $\mathcal{H}$ be the space of all holomorphic functions $f(z)=\sum_{m=0}^{\infty} f_{m} z^{m}$ on the unit disc $\mathbf{D} \subset \mathbf{C}$ for which

$$
\|f\|_{\mathcal{H}}^{2}:=\frac{1}{2} \sum_{m=0}^{\infty}(m+1)(m+2)\left|f_{m}\right|^{2}<\infty .
$$

With the scalar product obtained by polarization, $\mathcal{H}$ has the reproducing kernel

$$
\begin{aligned}
K(z, w) & =\sum_{m=0}^{\infty} \frac{2 z^{m} \bar{w}^{m}}{(m+1)(m+2)} \\
& =2 \frac{t+(1-t) \log (1-t)}{t^{2}}, \quad t:=z \bar{w} .
\end{aligned}
$$

Let $T \in \mathcal{B}(\mathcal{H})$ be the orthogonal projection in $\mathcal{H}$ onto the constants:

$$
T=\langle\cdot, \mathbf{1}\rangle \mathbf{1},
$$

so that

A calculation shows that

$$
\widetilde{T}(z)=\frac{1}{K(z, z)}
$$

$$
\begin{aligned}
& \|\partial \partial \widetilde{T}\|^{2}=\left|\frac{\widetilde{T}_{111}}{g_{1 \overline{1}}}\right|^{2}= \\
& \left|\frac{-t(t+(1-t) \log (1-t))\left[t^{2}(2+t)+(4-3 t) t \log (1-t)+2(1-t)^{2} \log ^{2}(1-t)\right]}{2\left(t^{2}-(1-t) \log ^{2}(1-t)\right)^{2}}\right|^{2}
\end{aligned}
$$

with $t:=|z|^{2}$, which tends to infinity as $|z| \nearrow 1$.

It can be shown that the scalar curvature $S$ equals 4 at the origin, and also increases with $|z|$ to infinity as $|z| \nearrow 1$.

The space $\mathcal{H}$ in the last example is a Dirichlet-type space. We do not know if there is an example of the form $\mathcal{H}=A^{2}(\Omega, w)$ for some domain $\Omega$ and positive weight function $w$ (i.e. a weighted Bergman space).

\section{Higher-ORDER DERIVATIVES}

So far, we have never really used the fact that norms of tensors - in particular, of the covariant derivatives of $\widetilde{T}-$ are scalar quantities, i.e. independent of the change of coordinate system. To get an analogue of Corollary 10 for $\nabla^{m} \widetilde{T}$ with arbitrary $m$, it turns out to be convenient to use such a coordinate change.

Fix a point $z_{0} \in \Omega$; for ease of notation, we will assume that $z_{0}=0$. Then for any $M \geq 1$, there exists a normal (other names: Bochner, geodesic) coordinate system of order $M$ at 0 , in which

$$
g_{j \bar{k}}(0)=g^{\bar{k} j}(0)=\delta_{j k}, \quad g_{j \bar{k}_{1} \ldots \bar{k}_{m}}(0)=g_{j_{1} \ldots j_{m} \bar{k}}(0)=0 \text { if } 1<m \leq M .
$$


For a proof of existence of normal coordinate systems of any order for an arbitrary Kähler metric, see e.g. [16, Lemma 3.7.1, or [7, Chapter VIII. Note that in terms of $L(z, z)=\log K(z, z)$, (28) becomes

$$
L_{j \bar{k}}(0,0)=\delta_{j k}, \quad L_{j \bar{k}_{1} \ldots \bar{k}_{m}}(0)=L_{j_{1} \ldots j_{m} \bar{k}}(0)=0 \text { if } 1<m \leq M .
$$

Differentiating the relation (17), we see that $g_{k \bar{l}} \partial_{i} g^{\bar{j} k}=-g^{\bar{j} k} g_{k \bar{l}}$, or

$$
\partial_{i} g^{\bar{j} p}=-g^{\bar{l} p} g^{\bar{j} k} g_{k \bar{l}} ;
$$

a similar result holds for $\bar{\partial}_{i} g^{\bar{j} p}$. From the definition of the covariant derivative and the formulas for the Riemann-Christoffel symbols, it thus follows by the Leibniz rule that the covariant derivatives of the curvature tensor $R_{i \bar{j} k \bar{l}}=g_{i \bar{j} k \bar{l}}-g^{\bar{q} p} g_{i k \bar{q}} g_{\bar{j} \bar{l} p}$ can be expressed as

$$
R_{i \bar{j} k \bar{l} / \alpha_{1} \ldots \alpha_{m}}=g_{i \bar{j} k \bar{l} \alpha_{1} \ldots \alpha_{m}}+\text { (a polynomial in } g^{\bar{\gamma}_{1} \gamma_{2}} \text { and } g_{\beta_{1} \ldots \beta_{s}}, 3 \leq s \leq m+3 \text { ). }
$$

A simple recursion argument then implies that

$$
\begin{array}{r}
g_{i \bar{j} k \bar{l} \alpha_{1} \ldots \alpha_{m}}=R_{i \bar{j} k \bar{l} \alpha_{1} \ldots \alpha_{m}}+\left(\text { a polynomial in } g^{\bar{\gamma}_{1} \gamma_{2}}, g_{\delta_{1} \delta_{2} \delta_{3}}\right. \text { and } \\
\left.R_{\beta_{1} \beta_{2} \beta_{3} \beta_{4} / \beta_{5} \ldots \beta_{s}} \text { with } s \leq m+3\right) .
\end{array}
$$

Consequently, in a normal coordinate system, we have in addition to (28),

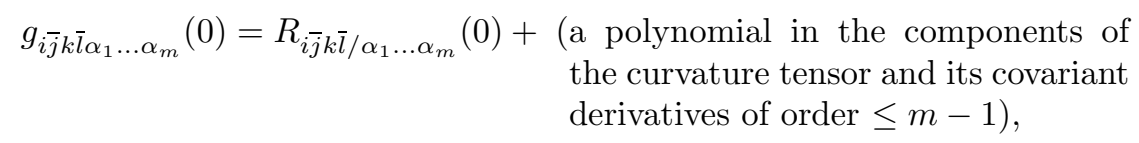

for any $m \leq M-4$.

After these preparations, we can prove the main result of this section.

Theorem 12. For any $m \geq 2$, there exists a scalar quantity $r_{m}$ on $\Omega$, given by a polynomial expression involving the contravariant metric tensor $g^{\bar{j} k}$, the curvature tensor $R_{i \bar{j} k \bar{l}}$, and the latter's covariant derivatives of orders $\leq 2 m-4$, such that

$$
\left\|\nabla^{m} \widetilde{T}(z)\right\|_{z}^{2} \leq r_{m}(z)\|T\|^{2}
$$

for any $z \in \Omega$ and $T \in \mathcal{B}(\mathcal{H})$.

Proof. Fix $z_{0} \in \Omega$ and pass to a normal coordinate system around $z_{0}=0$ of order $M \geq 2 m$, as above. By (28) and (31), we will thus have

$$
\begin{aligned}
& L_{i \bar{j}}(0,0)=\delta_{i j}, \quad L_{i \bar{j} k_{1} \ldots k_{p}}(0,0)=L_{i \bar{j} \bar{k}_{1} \ldots \bar{k}_{p}}(0,0)=0, \\
& L_{i \bar{j} k \bar{l} \alpha_{1} \ldots \alpha_{p}}(0,0)=R_{i \bar{j} k \bar{l} / \alpha_{1} \ldots \alpha_{p}}(0)+\left(\text { a polynomial in } \nabla^{s} R, s \leq p-1\right),
\end{aligned}
$$

for all $p \leq 2 m-4$.

If $\phi$ is any holomorphic function on $\Omega$ with $\phi(0) \neq 0$, then the multiplication operator $M_{\phi}: f \mapsto \phi f$ is a unitary isomorphism of the Hilbert space $\mathcal{H}$ onto another Hilbert space, say, $\mathcal{H}^{\prime} \subset \mathcal{O}(\Omega)$, whose reproducing kernel is given by

$$
K^{\prime}(x, y)=\phi(x) \overline{\phi(y)} K(x, y) .
$$

It follows that the Berezin transform (in $\mathcal{H}$ ) of an operator $T \in \mathcal{B}(\mathcal{H}$ ) is equal to the Berezin transform (in $\mathcal{H}^{\prime}$ ) of the operator $T^{\prime}:=M_{\phi} T M_{\phi}^{-1} \in \mathcal{B}\left(\mathcal{H}^{\prime}\right)$, and $\|T\|_{\mathcal{H} \rightarrow \mathcal{H}}=\left\|T^{\prime}\right\|_{\mathcal{H}^{\prime} \rightarrow \mathcal{H}^{\prime}}$. Since $L^{\prime}(x, y)=L(x, y)+\log \phi(x)+\overline{\log \phi(y)}$, taking $\phi(x)=$ $\sqrt{K(0,0)} / K(x, 0)$ we can achieve upon passing from $L$ to $L^{\prime}$ that

$$
L_{j_{1} \ldots j_{p}}(0,0)=L_{\bar{j}_{1} \ldots \bar{j}_{p}}(0,0)=0 \quad \forall p \geq 0 .
$$


Note that since $L_{j \bar{k}}^{\prime}=L_{j \bar{k}}$, (32) remains in force upon passage from $L$ to $L^{\prime}$. From now on, we will therefore assume that both (32) and (33) hold.

As before,

$$
\left|\widetilde{T}_{/ \alpha_{1} \ldots \alpha_{m}}\right|=\left|\operatorname{tr}\left(T A_{/ \alpha_{1} \ldots \alpha_{m}}\right)\right| \leq\|T\|\left\|A_{/ \alpha_{1} \ldots \alpha_{m}}\right\|_{\operatorname{tr}} .
$$

Using again the definition of covariant derivative, the formulas for the RiemannChristoffel symbols, and (30), we see that

$$
A_{/ \alpha_{1} \ldots \alpha_{m}}=\sum_{p \leq m} \sum_{\boldsymbol{\beta}=\left(\beta_{1}, \ldots, \beta_{p}\right)} P_{\boldsymbol{\alpha} \boldsymbol{\beta}} D^{\boldsymbol{\beta}} A
$$

with $D^{\boldsymbol{\beta}}=D_{\beta_{1}} \ldots D_{\beta_{p}}$, where $D_{\beta}=\partial_{j}$ if $\beta=j$ and $=\bar{\partial}_{j}$ if $\beta=\bar{j}$, and $P_{\boldsymbol{\alpha} \boldsymbol{\beta}}$ is a polynomial, depending only on $\boldsymbol{\alpha}:=\left(\alpha_{1}, \ldots, \alpha_{m}\right)$ and $\boldsymbol{\beta}$, in $g^{\bar{j} k}$ and $g_{\gamma_{1} \ldots \gamma_{s}}$ with $2 \leq s \leq m+1$.

Next, applying the Leibniz rule to $A(z)=K(z, z)^{-1}\left\langle\cdot, K_{z}\right\rangle K_{z}$ shows that

$$
\begin{aligned}
D^{\boldsymbol{\beta}} A(z) & =\sum_{\boldsymbol{\gamma} \cup \boldsymbol{\delta} \cup \boldsymbol{\epsilon}=\boldsymbol{\beta}} c_{\boldsymbol{\beta} \boldsymbol{\gamma} \boldsymbol{\delta} \boldsymbol{\epsilon}} D^{\boldsymbol{\gamma}} K(z, z)^{-1}\left\langle\cdot, D_{z}^{\overline{\boldsymbol{\delta}}} K_{z}\right\rangle D_{z}^{\boldsymbol{\epsilon}} K_{z} \\
& \equiv \sum_{\boldsymbol{\gamma} \cup \boldsymbol{\delta} \cup \boldsymbol{\epsilon}=\boldsymbol{\beta}} c_{\boldsymbol{\beta} \boldsymbol{\gamma} \boldsymbol{\delta} \boldsymbol{\epsilon}} D^{\boldsymbol{\gamma}} K(z, z)^{-1} \mathbf{X}_{z}^{\boldsymbol{\delta} \boldsymbol{\epsilon}},
\end{aligned}
$$

where the sum extends over all disjoint partitions of the index set $\beta_{1}, \ldots, \beta_{p}$ into subsets $\boldsymbol{\gamma}, \boldsymbol{\delta}, \boldsymbol{\epsilon}$, with $\overline{\boldsymbol{\delta}}$ and $\boldsymbol{\epsilon}$ containing only barred indices; here $c_{\boldsymbol{\alpha} \boldsymbol{\beta} \boldsymbol{\gamma} \boldsymbol{\delta}}$ are constants depending only on the subscripts indicated, and the subscript $z$ in $D_{z}^{\bar{\delta}}, D_{z}^{\epsilon}$ is supplied in order to make it clear what is the differentiated variable.

The last two formulas show that $A_{/ \alpha_{1} \ldots \alpha_{m}}$ is an operator of rank at most $N_{m, n}$, the number of multi-indices $\left(\delta_{1}, \ldots, \delta_{n}\right)$ of length $\delta_{1}+\cdots+\delta_{n} \leq m$. Clearly, $N_{m, n} \leq(m+1)^{n}$.

Now if $F$ is any operator of rank $r$ and $\lambda_{1}, \ldots, \lambda_{r}$ are the nonzero eigenvalues of $\left(F^{*} F\right)^{1 / 2}$, then

$$
\|F\|_{\mathrm{tr}}^{2}=\left(\sum_{j} \lambda_{j}\right)^{2} \leq r \sum_{j} \lambda_{j}^{2}=r \operatorname{tr}\left(F^{*} F\right)
$$

Consequently,

$$
\left\|A_{/ \alpha_{1} \ldots \alpha_{m}}\right\|_{\mathrm{tr}}^{2} \leq N_{m, n} \operatorname{tr}\left(A_{/ \alpha_{1} \ldots \alpha_{m}}^{*} A_{/ \alpha_{1} \ldots \alpha_{m}}\right) .
$$

Finally,

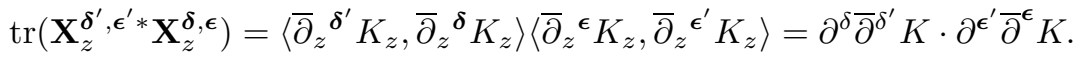

Let us now switch temporarily to the normal coordinate system with (32) and (33). Since $g^{\bar{j} k}(0)=\delta_{j k}$, we have

$$
\begin{aligned}
\left\|\nabla^{m} \widetilde{T}(0)\right\|^{2} & =2^{m} \sum_{\alpha_{1}, \ldots, \alpha_{m}}\left|\widetilde{T}_{/ \alpha_{1} \ldots \alpha_{m}}(0)\right|^{2} \\
& \leq 2^{m}\|T\|^{2} \sum_{\alpha_{1}, \ldots, \alpha_{m}}\left\|A_{/ \alpha_{1} \ldots \alpha_{m}}(0)\right\|_{\mathrm{tr}}^{2} \quad \text { by (134) } \\
& \leq 2^{m} N_{m, n}\|T\|^{2} \sum_{\alpha_{1}, \ldots, \alpha_{m}} \operatorname{tr}\left(A_{/ \alpha_{1} \ldots \alpha_{m}}^{*}(0) A_{/ \alpha_{1} \ldots \alpha_{m}}(0)\right) \quad \text { by (37) } \\
& \leq 2^{m} N_{m, n}\|T\|^{2} g^{\bar{\alpha}_{1}^{\prime} \alpha_{1}}(0) \ldots g^{\bar{\alpha}_{m}^{\prime} \alpha_{m}}(0) \operatorname{tr}\left(A_{/ \alpha_{1}^{\prime} \ldots \alpha_{m}^{\prime}}^{*}(0) A_{/ \alpha_{1} \ldots \alpha_{m}}(0)\right) .
\end{aligned}
$$


Since both the left-hand side and the ultimate right-hand side are scalar quantities, it follows that

$$
\left\|\nabla^{m} \widetilde{T}\right\|^{2} \leq 2^{m} N_{m, n}\|T\|^{2} g^{\bar{\alpha}_{1}^{\prime} \alpha_{1}} \ldots g^{\bar{\alpha}_{m}^{\prime} \alpha_{m}} \operatorname{tr}\left(A_{/ \alpha_{1}^{\prime} \ldots \alpha_{m}^{\prime}}^{*} A_{/ \alpha_{1} \ldots \alpha_{m}}\right)
$$

holds in any coordinate system (not necessarily normal). Furthermore, from (35), (36) and (38),

$$
\begin{aligned}
& \operatorname{tr}\left(A_{/ \alpha_{1}^{\prime} \ldots \alpha_{m}^{\prime}}^{*} A_{/ \alpha_{1} \ldots \alpha_{m}}\right)=\sum_{p, p^{\prime} \leq m} \sum_{\begin{array}{c}
\boldsymbol{\beta}=\left(\beta_{1}, \ldots, \beta_{p}\right) \\
\boldsymbol{\beta}^{\prime}=\left(\beta_{1}^{\prime}, \ldots, \boldsymbol{\beta}_{p^{\prime}}\right)
\end{array}} P_{\boldsymbol{\alpha} \boldsymbol{\beta}} \overline{P_{\boldsymbol{\alpha}^{\prime} \boldsymbol{\beta}^{\prime}}} \\
& \times \sum_{\substack{\gamma \cup \boldsymbol{\delta} \cup \boldsymbol{\epsilon}=\boldsymbol{\beta} \\
\boldsymbol{\gamma}^{\prime} \cup \boldsymbol{\delta}^{\prime} \cup \boldsymbol{\epsilon}^{\prime}=\boldsymbol{\beta}^{\prime}}} c_{\boldsymbol{\beta} \boldsymbol{\gamma} \boldsymbol{\delta} \boldsymbol{\epsilon}} \overline{c_{\boldsymbol{\beta}^{\prime} \boldsymbol{\gamma}^{\prime} \boldsymbol{\delta}^{\prime} \boldsymbol{\epsilon}^{\prime}}} D^{\gamma}\left(K^{-1}\right) \cdot D^{\boldsymbol{\gamma}^{\prime}}\left(K^{-1}\right) \cdot \partial^{\delta} \bar{\partial}^{\delta^{\prime}} K \cdot \partial^{\epsilon^{\prime}} \bar{\partial}^{\epsilon} K .
\end{aligned}
$$

Using the formula

$$
\frac{D^{\boldsymbol{\theta}} K}{K}=e^{-L} D^{\boldsymbol{\theta}} e^{L}=\sum_{\boldsymbol{\theta}=\boldsymbol{\theta}_{1}+\boldsymbol{\theta}_{2}+\ldots} L_{\boldsymbol{\theta}_{1}} L_{\boldsymbol{\theta}_{2}} \ldots
$$

where the summation extends over all disjoint partitions of the multi-index $\boldsymbol{\theta}$ into nonempty sub-multi-indices $\boldsymbol{\theta}_{1}, \boldsymbol{\theta}_{2}, \ldots$, and similarly for $K D^{\boldsymbol{\theta}}\left(K^{-1}\right)=e^{L} D^{\boldsymbol{\theta}} e^{-L}$, we see that

$$
D^{\gamma}\left(K^{-1}\right) \cdot D^{\gamma^{\prime}}\left(K^{-1}\right) \cdot \partial^{\delta} \bar{\partial}^{\delta^{\prime}} K \cdot \partial^{\epsilon^{\prime}} \bar{\partial}^{\epsilon} K=\mathcal{P}_{\boldsymbol{\gamma} \gamma^{\prime} \delta \delta^{\prime} \epsilon \epsilon^{\prime}}
$$

where $\mathcal{P}_{\boldsymbol{\gamma} \gamma^{\prime} \delta \delta^{\prime} \epsilon \epsilon^{\prime}}$ is a polynomial in the derivatives of $L$ of orders $\leq 2 m$, whose coefficients depend only on $\boldsymbol{\gamma}, \boldsymbol{\gamma}^{\prime}, \boldsymbol{\delta}, \boldsymbol{\delta}^{\prime}, \boldsymbol{\epsilon}, \boldsymbol{\epsilon}^{\prime}$ and $n$. Now evaluating everything at $z_{0}=0$ and using (32) and (33), we obtain

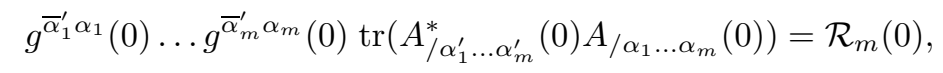

where $\mathcal{R}_{m}$ is a polynomial, whose coefficients depend only on $m$ and $n$, in $g^{\bar{j} k}$, $R_{i \bar{j} k \bar{l}}$, and the covariant derivatives of $R_{i \bar{j} k \bar{l}}$ of orders $\leq 2 m-4$ at $z_{0}=0$. Since the left-hand side is a scalar quantity, it follows again that $\mathcal{R}_{m}$ must also be of the indicated form in any other (i.e. not necessarily normal) coordinate system. Inserting this into (39) and setting $r_{m}=N_{m, n} \mathcal{R}_{m}$, the assertion of the theorem follows.

\section{Concluding Remarks}

For bounded strictly-pseudoconvex domains $\Omega \subset \mathbf{C}^{n}$ with smooth boundary, the scalar curvature of the Bergman metric is known to tend to $n$ at the boundary [21] ; thus $S$ has a finite upper bound on $\Omega$. By Corollary 10, it follows that for these domains with the Bergman metric, the covariant differentiation conjecture (5) is true for $k=2$. Since the exact formulas for $r_{k}$ with $k \geq 3$ are not available (nor is - to the authors' knowledge - very much known about the boundary behaviour of the covariant derivatives of the curvature tensor of the Bergman metric), it is still possible that the conjecture holds for the Bergman metric on such domains.

\footnotetext{
${ }^{3}$ More precisely, 21] deals with the holomorphic sectional curvature $\rho(z, X)$ (defined further below) rather than $S$; the latter can be obtained (with the normalizations for $\rho$ and $S$ used in this paper, which differ from those in 21] or 30] by factors of 2 and -2 , respectively, and from those in [23] by sign) as $-n(n+1) / 2$ times the average of the former over all $X$ in the unit sphere. See also [23] and 6] for later generalizations to some weakly pseudoconvex domains.
} 
Note that our estimate for the scalar curvature

$$
S \geq-n(n+1)
$$

seems to be new even for the weighted Bergman metrics.

Taking in the formula (25) $v^{j m}=u^{j} u^{m}$ for $u=\left(u^{j}\right) \in \mathbf{C}^{n}$, we get

$$
|\partial \partial \widetilde{T}(u, u)|^{2} \equiv\left|u^{j} u^{m} \widetilde{T}_{/ j m}\right|^{2} \leq\|T\|^{2}\left(R_{j \bar{k} l \bar{m}} u^{j} \bar{u}^{k} u^{l} \bar{u}^{m}+2\|u\|_{z}^{4}\right) .
$$

The quantity

$$
\rho(z, u):=-\frac{R_{j \bar{k} l \bar{m}}(z) u^{j} \bar{u}^{k} u^{l} \bar{u}^{m}}{\|u\|_{z}^{4}}
$$

is known as the holomorphic sectional curvature 4 of the metric (1) at $z$ in the direction $u$; (40) thus shows that $\rho(z, u) \leq 2$, a result known for $\mathcal{H}=A^{2}(\Omega)$ since Bergman [5]. Similarly one can get from (40) that the bisectional curvature

$$
B(z ; u, w):=-\frac{R_{j \bar{k} l \bar{m}}(z) u^{j} \bar{u}^{k} w^{l} \bar{w}^{m}}{\|u\|_{z}^{2}\|w\|_{z}^{2}}
$$

is bounded from above by 2 as well, again for any $\mathcal{H} \subset \mathcal{O}(\Omega)$.

Going through the proof of Theorem 8 , one can check that the estimate $\|\partial \partial \widetilde{T}\|^{2} \leq$ $\left(S+n^{2}+n\right)\|T\|^{2}$ is sharp for $n=1$, while for $n \geq 2$ there is obviously some room left as a result of estimating the operator norm by the trace norm. A similar comment applies to the estimate $\|\partial \bar{\partial} \widetilde{T}\|^{2} \leq 4 n\|T\|^{2}$ in Theorem 9. Getting better (if not sharp) estimates for $n \geq 2$ would be of considerable interest.

If $f \in \mathcal{O}(\Omega)$ is a multiplier for $\mathcal{H}$, i.e. $f h \in \mathcal{H}$ for all $h \in \mathcal{H}$, then the multiplication operator $M_{f}: h \mapsto f h$ is bounded on $\mathcal{H}$ and $\widetilde{M_{f}}=f$. Thus Theorems 7 and 8 yield

$$
\|\partial f(z)\|_{z} \leq\left\|M_{f}\right\|, \quad\|\partial \partial f(z)\|_{z} \leq \sqrt{S+n^{2}+n}\left\|M_{f}\right\| .
$$

In particular, for $\mathcal{H}=A^{2}(\Omega, w)$ the weighted Bergman space on a bounded domain $\Omega$ with respect to an integrable positive weight $w$, any bounded holomorphic function $f \in H^{\infty}(\Omega)$ is a multiplier, with $\left\|M_{f}\right\| \leq\|f\|_{\infty}$, and hence

$$
\|\partial f(z)\|_{z} \leq\|f\|_{\infty} \quad \forall z \in \Omega
$$

and similarly for $\|\partial \partial f\|$. The holomorphic functions $f$ satisfying

$$
\|f\|_{\mathrm{Bl}}:=\sup _{z \in \Omega}\|\partial f(z)\|_{z}<\infty
$$

form the Bloch space $\mathrm{Bl}(\Omega)$ of Timoney [29]. As in [13, (41) thus shows that $H^{\infty}(\Omega) \subset \operatorname{Bl}(\Omega)$ continuously, a result proved for $\mathcal{H}=A^{2}(\Omega)$ by Hahn $[18$.

Following $\left[13\right.$, one can also define the real-analytic Bloch space $\mathrm{Bl}^{\omega}(\Omega)$ to consist of all real-analytic functions on $\Omega$ satisfying

$$
\sup _{z \in \Omega}\|\nabla f(z)\|_{z}<\infty .
$$

Theorem 7 thus says that $\widetilde{T}$ belongs to $\mathrm{Bl}^{\omega}(\Omega)$, for any $T \in \mathcal{B}(\mathcal{H})$.

For $\mathcal{H}=A^{2}(\mathbf{D})$, the Bergman space on the unit disc, and $f$ holomorphic, (42) is actually known to be equivalent to

$$
\sup _{z \in \Omega}\|\partial \partial f(z)\|_{z}<\infty
$$

\footnotetext{
${ }^{4}$ See e.g. [30], $\$ 7.5$, page 177 .
} 
and similarly for $\left\|\partial^{k} f\right\|, k>2$. It does not seem to be known whether this equivalence prevails for all bounded strictly-pseudoconvex domains in $\mathbf{C}^{n}$ with smooth boundary. For bounded symmetric domains, or other domains with nonsmooth boundaries (including the polydisc), the situation is more complicated; see [1].

Although the authors made no effort to proceed in this direction, it is highly likely that most (if not all) of the results in this paper also remain in force for reproducing kernel Hilbert spaces $\mathcal{H}$ of holomorphic sections of line bundles over complex manifolds $\Omega$. See Peetre 26] for the definition of the Berezin transform in this context.

\section{REFERENCES}

[1] J. Arazy, M. Engliš: $Q_{p}$-spaces on bounded symmetric domains, J. Funct. Spaces Appl. 6 (2008), 1419-1457. MR2444523

[2] H. Bateman, A. Erdélyi, Higher transcendental functions, vol. III, McGraw-Hill, New YorkToronto-London, 1953. MR0066496 (16:586c)

[3] M. Beals, C. Fefferman, and R. Grossman: Strictly pseudoconvex domains in $\mathbf{C}^{n}$, Bull. Amer. Math. Soc. 8(1983), 125-326. MR684898 (85a:32025)

[4] F.A. Berezin: Quantization, Math. USSR Izvestiya 8 (1974), 1109-1163. MR0395610 $(52: 16404)$

[5] S. Bergman, The kernel function and conformal mapping. Second, revised edition, Mathematical Surveys, vol. 5, American Mathematical Society, Providence, 1970. MR0507701 $(58: 22502)$

[6] H. Boas, E. Straube, J. Yu: Boundary limits of the Bergman kernel and metric, Michigan Math. J. 42 (1995), 449-461. MR.1357618 (96j:32029)

[7] S. Bochner, K. Yano, Curvature and Betti numbers, Princeton University Press, Princeton, 1953. MR0062505 (15:989f)

[8] H. Bommier-Hato: Lipschitz estimates for the Berezin transform, J. Funct. Spaces Appl. 8 (2010), 103-128. MR2667872

[9] H. Bommier-Hato: Derivatives of the Berezin transform, preprint (2008).

[10] H. Bommier-Hato, E.H. Youssfi: Hankel operators on weighted Fock spaces, Integ. Eqs. Oper. Theory 59 (2007), 1-17. MR2351271 (2008k:47064)

[11] L.A. Coburn: A Lipschitz estimate for Berezin's operator calculus, Proc. Amer. Math. Soc. 133 (2005), 127-131. MR2085161 (2005e:47060)

[12] L.A. Coburn: Sharp Berezin-Lipschitz estimates, Proc. Amer. Math. Soc. 135 (2007), 11631168. MR2262921 (2007g:47033)

[13] L.A. Coburn, B. Li: Directional derivative estimates for Berezin's operator calculus, Proc. Amer. Math. Soc. 136 (2008), 641-649. MR2358506 (2008h:47048)

[14] M. Engliš: A characterization of symmetric domains, J. Math. Kyoto Univ. 46 (2006), 123146. MR.2260820 (2008c:32031)

[15] M. Engliš, G. Zhang: On the derivatives of the Berezin transform, Proc. Amer. Math. Soc. 134 (2006), 2285-2294. MR2213701 (2007c:47029)

[16] P.B. Gilkey, Invariance theory, the heat equation, and the Atiyah-Singer index theorem, Publish or Perish, 1984. MR783634 (86j:58144)

[17] I.C. Gohberg, M.G. Krein, Introduction to the theory of linear nonselfadjoint operators, Translations of Mathematical Monographs, vol. 18, Amer. Math. Soc., Providence, 1969. MR0246142 (39:7447)

[18] K.T. Hahn: On completeness of the Bergman metric and its subordinate metric, Proc. Nat. Acad. Sci. USA 73 (1976), 4294. MR0417459 (54:5509)

[19] S. Helgason, Differential geometry and symmetric spaces, Academic Press, New York-London, 1962. MR0145455 (26:2986)

[20] M. Jarnicki, P. Pflug, Invariant distances and metrics in complex analysis, Walter de Gruyter, 1993. MR $1242120(94 \mathrm{k}: 32039)$

[21] P.F. Klembeck: Kähler metrics of negative curvature, the Bergman metric near the boundary, and the Kobayashi metric on smooth bounded strictly pseudoconvex sets, Indiana Univ. Math. J. 27 (1978), 275-282. MR0463506 (57:3455) 
[22] S. Kobayashi, K. Nomizu, Foundations of differential geometry, vol. II, Interscience, New York -London-Sydney, 1969. MR0238225 (38:6501)

[23] S.G. Krantz, J. Yu: On the Bergman invariant and curvatures of the Bergman metric, Illinois J. Math. 40 (1996), 226-244. MR.1398092 (97g:32026)

[24] T. Mazur, P. Pflug, M. Skwarczynski: Invariant distances related to the Bergman kernel function, Proc. Amer. Math. Soc. 94 (1985), 72-76. MR781059 (86i:32047)

[25] K. Nam, D. Zheng, C. Zhong: m-Berezin transform and compact operators, Rev. Mat. Iberoam. 22 (2006), 867-892. MR2320405 (2008d:47059)

[26] J. Peetre: The Berezin transform and Ha-plitz operators, J. Operator Theory 24 (1990), 165-186. MR1086552 (91k:47058)

[27] T. Sakai, Riemannian geometry, Transl. Math. Monographs, vol. 149, Amer. Math. Soc., Providence, 1996. MR1390760 (97f:53001)

[28] D. Suárez: Approximation and symbolic calculus for Toeplitz algebras on the Bergman space, Rev. Mat. Iberoam. 20 (2004), 563-610. MR2073132 (2005e:47075)

[29] R.M. Timoney: Bloch functions in several complex variables I, Bull. London Math. Soc. 12 (1980), 241-267; II, J. Reine Angew. Math. 319 (1980), 1-22. MR576974 (83b:32004) MR0586111 (83b:32005)

[30] F. Zheng, Complex differential geometry, Amer. Math. Soc., Providence, 2000. MR:1777835 (2001i:32035)

Mathematics Institute, Silesian University in Opava, Na RybníčKu 1, 74601 Opava, Czech Republic - And - Mathematics Institute, Academy of Sciences, Žitná 25, 11567 Prague 1, Czech Republic

E-mail address: englis@math.cas.cz

Mathematics Institute, Silesian University in Opava, Na RybníčKu 1, 74601 Opava, Czech Republic

E-mail address: Renata.Otahalova@math.slu.cz 